\title{
Metastatic Squamous Cell Carcinoma from Lung Adenocarcinoma after Epidermal Growth Factor Receptor Tyrosine Kinase Inhibitor Therapy
}

\author{
Hyung Kyu Park · Youjeong Seo · Yoon-La Choi · Myung-Ju Ahn $•$ Joungho Han \\ Department of Pathology and Translational Genomics, 'Division of Hematology and Oncology, Department of Medicine, Samsung Medical Center, \\ Sungkyunkwan University School of Medicine, Seoul, Korea
}

Inhibition of mutated epidermal growth factor receptor (EGFR), EGFR tyrosine kinase inhibitor (TKI), is one of the most successful cancer targeted therapies. ${ }^{1}$ While this therapy has been beneficial for many non-small cell lung cancer (NSCLC) patients with activated EGFR mutations, almost all patients inevitably develop acquired resistance which limits the median response duration to around 1 year. ${ }^{2}$ Most of the mechanisms leading to EGFR TKI resistance are additional mutation or amplification of alternative pathways, and morphological transformation to small cell lung cancer (SCLC). ${ }^{3}$

Recently, several authors reported another type of morphologic transformation for EGFR TKI resistance, namely, transformation from adenocarcinoma to squamous cell carcinoma. ${ }^{4-6}$ However, the reports were of limited value because they were based only on biopsied specimens. This report describes a case of lung adenocarcinoma with transformation to squamous cell carcinoma that was established histological evaluation of lobectomy specimen.

\section{CASE REPORT}

A 40-year-old man with a 23-year smoking history received a medical check-up, which revealed a solitary pulmonary nodule. Chest computed tomography revealed a 17 -mm-sized tumor at

\section{Corresponding Author}

Joungho Han, MD

Department of Pathology and Translational Genomics, Samsung Medical Center, Sungkyunkwan University School of Medicine, 81 Irwon-ro, Gangnam-gu, Seoul 06351, Korea

Tel: +82-2-3410-2765, Fax: +82-2-3410-0025, E-mail: hanjho@skku.edu

Received: August 24, 2016 Revised: October 16, 2016 Accepted: October 17, 2016 the peripheral posterior segment of the right upper lobe (clinically T1aN0M0). The patient underwent a right upper lobectomy with mediastinal lymph node dissection. Histological examination showed a $1.3-\mathrm{cm}$-sized poorly differentiated adenocarcinoma with solid and acinar growth patterns with visceral pleural invasion and no lymph node metastasis (pT2aN0). The tumor cells showed marked nuclear atypia, numerous mitoses (23/10 high power field [HPF]) and multifocal microscopic necrosis. The tumor was dissected and entirely embedded. We morphologically and immunohistochemically evaluated all tumor sections to rule out the possibility of adenosqumous carcinoma (Fig. 1A-E). The patient received adjuvant chemotherapy with combined vinorelbine and cisplatin.

Four years later, multiple metastatic lesions were found at the right lower lobe, right pleura, right fifth rib, and right femoral head. EGFR mutation analysis, using a previously resected lobectomy specimen, revealed a deletion at exon 19 of the EGFR gene. The patient was treated with afatinib, which is an EGFR TKI and radiation therapy. The patient showed partial remission of the tumor.

Two years later, the patient developed multiple metastatic lesions throughout the body, including a telangiectatic nodule on the scalp. A punch biopsy was performed and histologic evaluation of the biopsied specimen showed a tumorous lesion in the deep dermis. This tumor showed squamoid appearance with sheet-like growth patterns and evident intercellular bridges. Compared to the previous lobectomy specimen, the tumor cells were more bland with moderate nuclear atypia, some mitotic activity (13/10 HPF) and no necrosis. Immunohistochemical staining showed diffuse and strong p63 immunoreactivity and no thyroid transcription factor 1 immunoreactivity (Fig. 1F-J). 


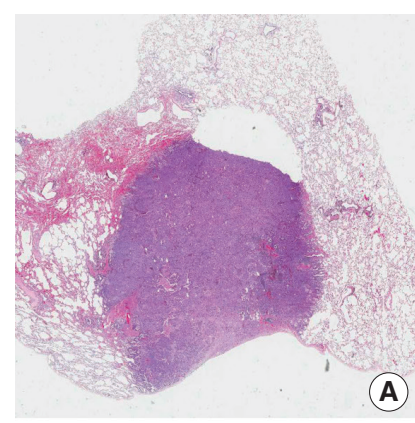

(A)
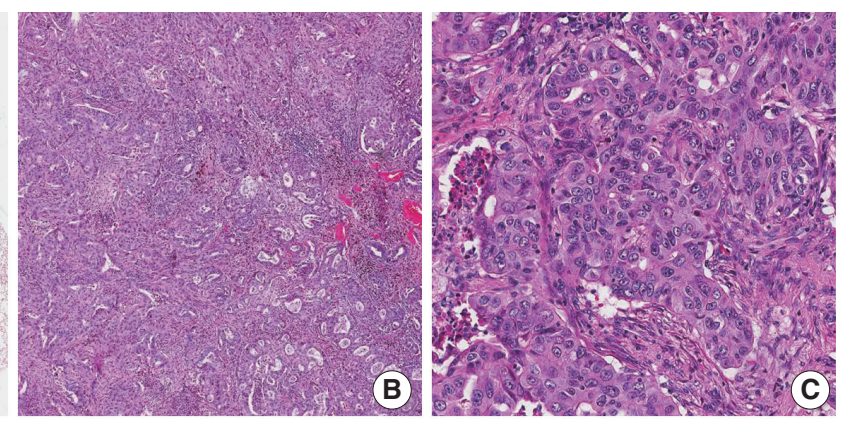

EGFR: deletion at the exon 19

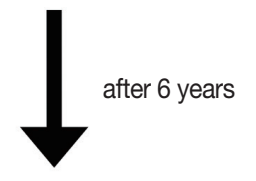

EGFR: deletion at the exon 19 and T790M point mutation
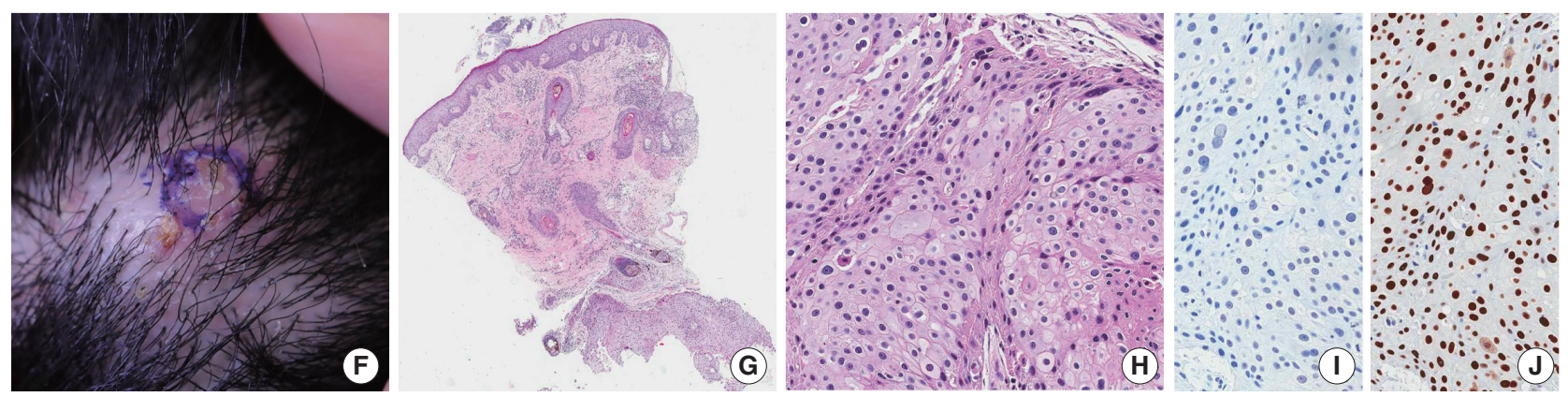

Fig. 1. Squamous metastasis from lung adenocarcinoma. Initial lobectomy specimen (A-E) and punch biopsy specimen of the scalp (F-J). (A-C) The well-demarcated adenocarcinoma with solid and acinar growth patterns was found in the lobectomy specimen. The tumor shows thyroid transcription factor 1 immunoreactivity (D) and no p63 immunoreactivity (E). Punch biopsy specimen of the telangiectatic nodule on scalp (F) shows a tumorous lesion at the deep dermis $(G)$. $(H)$ This tumor shows a squamoid appearance with a sheet-like growth pattern. The tumor shows no TTF-1 immunoreactivity (I) and diffuse p63 immunoreactivity (J). EGFR, epidermal growth factor receptor.

EGFR mutation analysis, using a biopsied specimen, revealed a deletion at exon 19 of the EGFR gene and an additional T790M point mutation. After several special and immunohistochemical stainings, this tumor was diagnosed as a metastatic carcinoma from the lung with squamous differentiation. The patient informed consent was waived by the Institutional Review Board of the Samsung Medical Center (2016-08-109).

\section{DISCUSSION}

Lung cancer is the most common cause of cancer deaths in Korea. ${ }^{7}$ Traditional therapy, including resection, platinum-based chemotherapy and radiation therapy, have only limited therapeutic value. Therefore, the 5 -year survival rate of lung cancer has not changed significantly in the past 30 years. ${ }^{8}$

EGFR TKI therapy, which specifically targets EGFR, was recently introduced and provided guidance in this situation. Targeting EGFR in patients with activating EGFR mutations has shown initial and significant success in practice. ${ }^{1}$ Unfortunately, the vast majority of patients develop resistance to the treatment, typically in less than 1 year. In this situation, understanding the mechanism of the resistance became very important.

Most of the mechanisms that lead to EGFR TKI resistance involve an additional mutation, such as a T790M mutation, or amplification of alternative pathways. In addition, morphological transformation is also a well-known mechanism. The most well known example of this interesting phenomenon is the transformation from NSCLC to SCLC?

Recently, several authors have described histologic transformation from lung adenocarcinoma to squamous cell carcinoma as a mechanism of resistance to EGFR TKI therapy. ${ }^{4-6}$ In four previously reported cases, all four patients were female and three of them had never been smokers. The most common EGFR mutation was an L858R point mutation and the most common acquired gene alteration was a T790M point mutation. All cases showed the same EGFR mutation in both primary 
and metastatic carcinomas.

The mechanism of this morphologic transformation is still poorly understood. Possible explanations include (1) small population of squamous cell carcinoma phenotype cells are already present before the EGFR TKI therapy and selectively survive during EGFR TKI therapy; or (2) carcinoma cells acquire a different morphologic phenotype during EGFR TKI therapy. ${ }^{6}$

Considering that primary adenosquamous carcinoma shows the same mutations in both adenocarcinoma and squamous cell carcinoma components, additional studies are needed and a solution is beyond the scope of this report.

In conclusion, lung primary adenocarcinoma can transform into squamous cell carcinoma after EGFR TKI therapy. We should be aware of this phenomenon to avoid misdiagnosis in practice.

\section{Conflicts of Interest}

No potential conflict of interest relevant to this article was reported.

\section{REFERENCES}

1. Stewart EL, Tan SZ, Liu G, Tsao MS. Known and putative mechanisms of resistance to EGFR targeted therapies in NSCLC patients with EGFR mutations-a review. Transl Lung Cancer Res 2015; 4: 67-81.
2. Choi CM, Lee JC. Mechanisms of acquired resistance to epidermal growth factor receptor inhibitors and overcoming strategies in lung cancer. J Lung Cancer 2012; 11: 59-65.

3. Yu HA, Arcila ME, Rekhtman N, et al. Analysis of tumor specimens at the time of acquired resistance to EGFR-TKI therapy in 155 patients with EGFR-mutant lung cancers. Clin Cancer Res 2013; 19: 2240-7.

4. Jukna A, Montanari G, Mengoli MC, et al. Squamous cell carcinoma "transformation" concurrent with secondary T790M mutation in resistant EGFR-mutated adenocarcinomas. J Thorac Oncol 2016; 11: e49-51.

5. Kuiper JL, Ronden MI, Becker A, et al. Transformation to a squamous cell carcinoma phenotype of an EGFR-mutated NSCLC patient after treatment with an EGFR-tyrosine kinase inhibitor. J Clin Pathol 2015; 68: 320-1.

6. Levin PA, Mayer M, Hoskin S, Sailors J, Oliver DH, Gerber DE. Histologic transformation from adenocarcinoma to squamous cell carcinoma as a mechanism of resistance to EGFR inhibition. J Thorac Oncol 2015; 10: e86-8.

7. Jung KW, Won YJ, Kong HJ, et al. Cancer statistics in Korea: incidence, mortality, survival, and prevalence in 2012. Cancer Res Treat 2015; 47: 127-41.

8. Cagle PT, Chirieac LR. Advances in treatment of lung cancer with targeted therapy. Arch Pathol Lab Med 2012; 136: 504-9.

9. Ahn S, Hwang SH, Han J, et al. Transformation to small cell lung cancer of pulmonary adenocarcinoma: clinicopathologic analysis of six cases. J Pathol Transl Med 2016; 50: 258-63. 